\title{
Analysis of Sleep Signals Based on Permutation Symbolic Transfer Entropy
}

\author{
Jun MIN, Jun WANG \\ ${ }^{1}$ Image Processing and Image Communications Key Lab,Nanjing University of Posts and \\ Telecommunications, China
}

${ }^{2}$ Smart Health Big Data Analysis and Location Services Engineering Lab of Jiangsu Province

Nanjing University of Posts and Telecommunications, China

wangi@njupt.edu.cn

\begin{abstract}
Keywords-Symbolic Transfer Entropy; Permutation Entropy; Sleep Signals
Abstract. In this paper, we use the method of permutation entropy symbolization to symbolize the original data. It mainly uses the size relation of the adjacent data to symbolize the original data, preserves the details of the original data, and overcomes the problem that global static symbolization method will lose the details of original data. In the paper, the symbolic transfer entropy based on permutation entropy symbolization is applied to the analysis of the sleep signal, and the method is compared with the method of symbolic transfer entropy based on quantization, and the following experimental results obtained are as follows: (1)The coupling of heart and brain in awake stage is stronger than that of first stage of non-rapid eye movement sleep. (2)Both in awake stage and first stage of non-rapid eye movement sleep, the coupling from brain to heart is stronger than the coupling from the heart to brain. (3)Using the symbolic transfer entropy method based on permutation entropy symbolization, we can get the conclusion (1) and the conclusion (2) for each data, and the method based on quantization can not fully derive the above conclusion, which is not applicable for some experimental data items. The results of the above experiments show that the symbolic transfer entropy method based on permutation entropy symbolization can obtain the better effect in the analysis of the coupling of EEG and ECG signal of sleep, which is more suitable for the clinical application.
\end{abstract}

\section{Introduction}

EEG signal and ECG signal are important means in the diagnosis of human physiology and clinical diseases. Attempts to analyze heart and brain signals have been the hotspots of domestic and foreign research, and they have high application value in clinical medicine. In recent years, in addition to the classic approach, a variety of new methods effectively promote the development of heart and brain signal analysis, there are: time-frequency analysis[1], artificial neural network[2], Nonlinear dynamics[3], independent component analysis[4]. The nonlinear dynamics method is gradually applied to the analysis of EEG and ECG signals.

The symbolic transfer entropy[5-6] serves as a parameter to quantify the degree of correlation of the two systems and can be used to process the finite length of the physiological signal data. Symbolic transfer entropy method based on quantization quantifies the data directly in the process of symbolization. This symbolic method uses static partitioning, although the dynamic characteristics are retained, the details are inevitably lost and the results are affected. The Permutation entropy[7-8] is an mean entropy parameter that measures the complexity of onedimensional time series. Its calculation is simple, and it is mainly uses the relation between the adjacent data to carry on the symbolization, and preserves the details of the original sequence and it can get very good results in practical application. Therefore, this paper uses the symbolic transfer entropy method based on permutation entropy symbolization to analyze the coupling of EEG and ECG signal in awake stage and first stage of non-rapid eye movement sleep, compare the effect with symbolic transfer entropy method based on quantization[9], the experimental results show that the symbolic transfer entropy method based on permutation entropy symbolization can preserve the details of the signal and get better effects in the analysis of sleeping signal. 


\section{Symbolic Transfer Entropy Based on Permutation Entropy Symbolization and Based on Quantization}

\subsection{Transfer entropy}

Transfer entropy is a parameter that quantifies the degree of correlation of two systems or the degree of chaos, which can be used to process finite length physiological signal data. The definition of transfer entropy was proposed by Schreiber in 2000:

$$
T_{J \rightarrow \mathrm{I}}=\sum p\left(i_{n+1}, i_{n}^{(k)}, j_{n}^{(l)}\right) \times \log \frac{\mathrm{p}\left(\left(i_{n+1} \mid i_{n}^{(k)}, j_{n}^{(l)}\right)\right.}{\mathrm{p}\left(\left(i_{n+1} \mid i_{n}^{(k)}\right)\right.}
$$

$i_{n}, j_{n}$ represents the states of the sequence I and Sequence $\mathrm{J}$ at the $\mathrm{n}$ moment. $i_{n}^{(k)}$ refers to a string of length $\mathrm{k}: i_{n-\mathrm{k}+1}, \ldots, i_{n}$. Similarly, $j_{n}^{(l)}$ is a string of length $1: j_{n-l+1}, \ldots, j_{n}$. Generally, $\mathrm{k}$ and 1 are one in order to facilitate computation.

\subsection{Symbolic transfer entropy based quantization}

Suppose that $x$ is the one-dimensional time series and the length is $N:\{x(i): 1 \leq i \leq N\}$.the symbolized sequence is $\mathrm{s}$, and the length is $\mathrm{N}:\{\mathrm{s}(\mathrm{i}): 1 \leq \mathrm{i} \leq \mathrm{N}\}$. The following is the calculation process of symbolic transfer entropy:

1).First, the time series $\mathrm{x}$ is symbolized, and the symbolic formula is as follows:

$$
\mathrm{s}(\mathrm{i})=\left\{\begin{array}{rr}
0 & u 1<x(i)<(1+\alpha) u 1 \text { or }(1+\alpha) \mathrm{u} 2<x(\mathrm{i})<u 2 \\
1 & (1+\alpha) u 1<x(i) \text { or } \mathrm{x}(\mathrm{i})<(1+\alpha) \mathrm{u} 2 \\
2 & (1-\alpha) u 1<x(i)<u 1 \text { or } \mathrm{u} 2<x(\mathrm{i})<(1-\alpha) \mathrm{u} 2 \\
3 & (1-\alpha) u 2<x(i)<(1-\alpha) u 1
\end{array}\right.
$$

$\mathrm{u} 1$ is the mean of all numbers greater than 0 in sequence $\mathrm{x}, \mathrm{u} 2$ is the mean of all numbers less than 0 in sequence $x$, and $\alpha$ is a constant, where we take the value of 0.05 .

(2)Secondly, the symbolic sequence s is encoded to obtain the sequence $\mathrm{w}$, the encoding is as follows:

$\mathrm{w}(\mathrm{i})=\mathrm{s}(\mathrm{i}) * 64+\mathrm{s}(\mathrm{i}+1) * 16+\mathrm{s}(\mathrm{i}+2) * 4+\mathrm{s}(\mathrm{i}+3)$;

for the encoded time series $\mathrm{w}$, the length is $\mathrm{N}:\{\mathrm{w}(\mathrm{i}): 1 \leq \mathrm{i} \leq \mathrm{N}\}$.

(3)In the phase space reconstruction, we set the embedding dimension $m=4$, and the time delay $\tau=1$.After phase space reconstruction, we get the matrix $X$, and each row of matrix is $\mathrm{X}(\mathrm{i})=[\mathrm{w}(\mathrm{i}), \mathrm{w}(\mathrm{i}+\tau), \ldots, \mathrm{w}(\mathrm{i}+(\mathrm{m}-1) \tau)]$;

(4)Finally, calculate the transfer entropy of the matrix X.

\subsection{Symbolic Transfer Entropy based on Permutation Entropy Symbolization}

Suppose that $\mathrm{x}$ is one-dimensional time series and the length is $\mathrm{N}:\{\mathrm{x}(\mathrm{i}): 1 \leq \mathrm{i} \leq \mathrm{N}\}$.

(1)Firstly, the phase space reconstruction is carried out to obtain the matrix X. In the phase space reconstruction process, set the embedding dimension $\mathrm{m}=4$, the time delay $\tau=1$ and $\tau=5$. After the phase space reconstruction, $\mathrm{X}$ is a matrix with row $\left(\mathrm{N}-(\mathrm{m}-1)^{*} \tau\right), \mathrm{X}(\mathrm{i})$ represent a row data of matrix: $X(i)=[x(i), x(i+\tau), \ldots, x(i+(m-1) * \tau)]$;

(2)Secondly, Each of row of the matrix $\mathrm{X}$ is symbolized by permutation entropy symbolization, and the symbolized matrix is $\mathrm{S}$.

(3)Finally, calculate the transfer entropy of matrix S.

\section{Symbolic Transfer Entropy Analysis of Sleep Signals}

The EEG and ECG signal data in awake stage and first stage of non-rapid eye movement sleep are taken from PhysioBank's MIT-BIH Polysomngraphic Database, the database records the multiparameter sleep data, including a guide EEG signal, a guide ECG signal, a guide EOG signal and other sleep signal, the basic data sampling rate is $250 \mathrm{~Hz}$, recording time up to $6 \mathrm{~h}$, with $30 \mathrm{~s}$ for a staging annotation information. In this paper, a total of 16 group of EEG and ECG sleep signal 
were selected, of which 8 groups of EEG and ECG sleep signals in awake stage, with corresponding 8 groups of EEG and ECG sleep signals in first stage of non-rapid eye movement sleep.

In this paper, we use the symbolic transfer entropy method based quantization and symbolic transfer entropy method based on permutation entropy symbolization to analyze the sleep signal in awake stage and first stage of non-rapid eye movement sleep. and compare this two method. The time delay $\tau=1$ of the symbolic transfer entropy based on quantization and the time delay $\tau=1$ and $\tau=5$ of the symbolic transfer entropy based on permutation entropy symbolization. In the process of solving symbolic transfer entropy, this paper uses following way to process the data, first of all, extract five sets of data from raw data, interception of data interval were:[51-1050],[10512050],[2051-3050],[3051-4050],[4051-5050]. The symbolic transfer entropy of the 5-segment data is obtained, and finally calculate the sum of the five symbolic transfer entropy, with the sum value to compare.

\subsection{Differences between the two methods in awake stage and in first stage of non-rapid eye movement sleep}
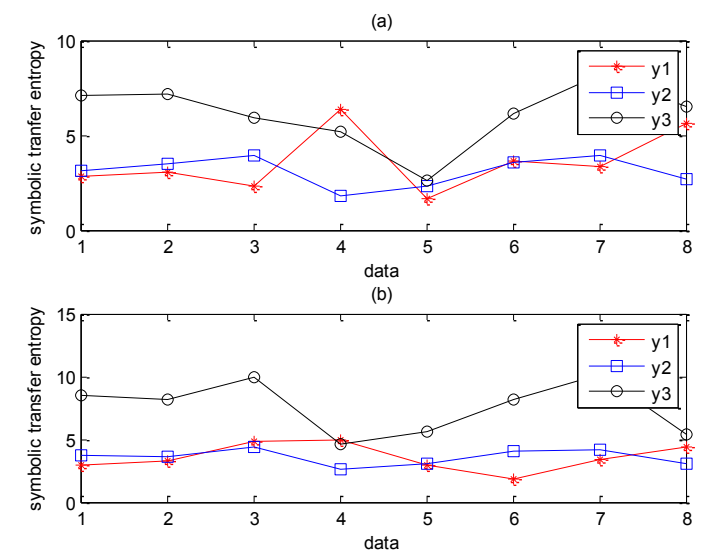

Fig. 1. The symbolic transfer entropy of various methods in awake stage.

Fig 1(a) is the symbol transfer entropy $\mathrm{T}_{\text {ecg } \rightarrow \text { eeg }}$ in awake stage and Fig 1(b) is the symbol transfer entropy $\mathrm{T}_{\text {eeg } \rightarrow \text { ecg }}$ in awake stage. In this figure, $\mathrm{y} 1$ is the value of the symbolic transfer entropy based on quantization, $\mathrm{y} 2$ is the value of symbolic transfer entropy based on the permutation entropy symbolization when $\tau=1$, and y 3 is when $\tau=5$. In awake stage, It can be seen from Fig 1 that the symbolic transfer entropy method based on permutation entropy symbolization is larger than symbolic transfer entropy based on quantization. At $\tau=5$, the symbolic transfer entropy method based on permutation entropy symbolization is larger than based on quantization. And at $\tau=1$, This two method are comparable, but the volatility of symbolic transfer entropy based on quantization is greater.
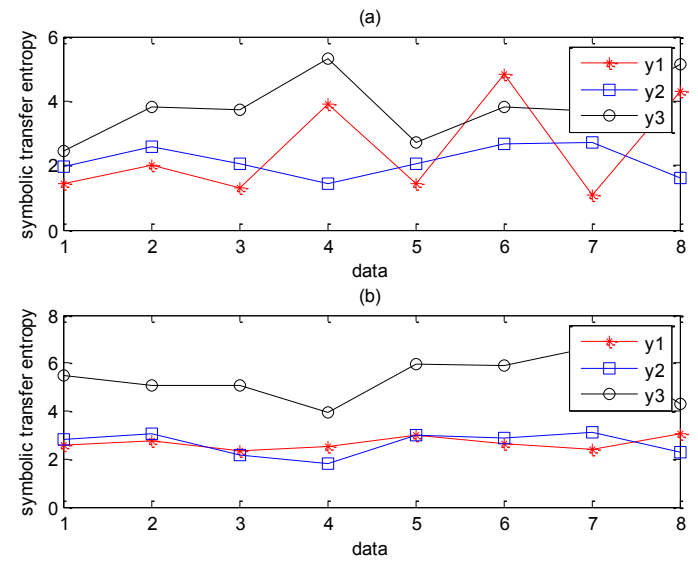

Fig. 2. The symbol transfer entropy of various methods in first stage of non-rapid eye movement sleep . 
Fig 2(a) is the symbol transfer entropy $\mathrm{T}_{\text {ecg } \rightarrow \text { eeg }}$ in first stage of non-rapid eye movement sleep and Fig 2(b) is the symbol transfer entropy $\mathrm{T}_{\text {eeg } \rightarrow \text { ecg }}$ in first stage of non-rapid eye movement sleep. In this figure, $\mathrm{y} 1$ is the value of the symbolic transfer entropy based on quantization, y2 is the value of symbolic transfer entropy based on the permutation entropy symbolization when $\tau=1$, and y 3 is when $\tau=5$. As can be seen from Fig 2, in first stage of non-rapid eye movement sleep, compare with symbolic transfer entropy based on quantization, symbolic transfer entropy based on permutation entropy symbolization is largest at $\tau=5$, and $\tau=1$ is almost equal to symbolic transfer entropy based quantization, but the fluctuation of the symbolic transfer entropy based on quantization is larger.

3.2The Symbolic transfer entropy based on permutation entropy symbolization $\boldsymbol{T}_{e c g \rightarrow e e g}$ and $\boldsymbol{T}_{e e g \rightarrow e c g}$ are compared between awake stage and first stage of non-rapid eye movement sleep.
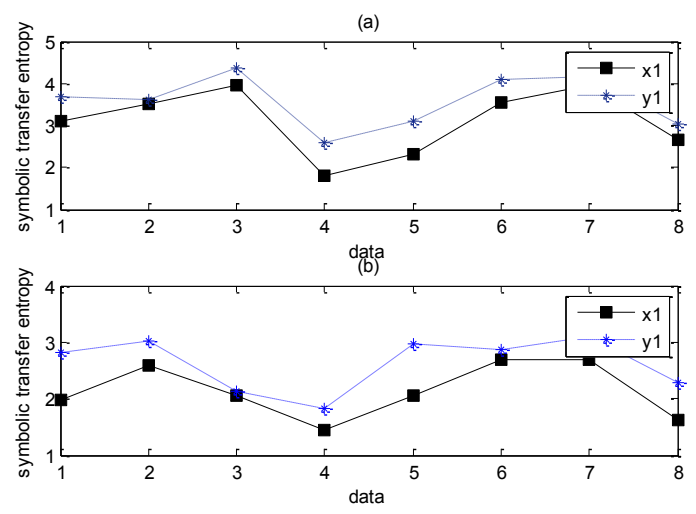

Fig. 3. Comparison of symbol transfer entropy based on permutation entropy symbolization

$$
\mathrm{T}_{\text {eeg } \rightarrow \text { ecg }} \text { and } \mathrm{T}_{\text {ecg } \rightarrow \text { eeg }} \text {. }
$$

Fig 3(a) is the comparison between $\mathrm{T}_{\text {eeg } \rightarrow \text { ecg }}$ and $\mathrm{T}_{\text {ecg } \rightarrow \text { eeg }}$ in awake stage and Fig3 (b) is the comparison $\mathrm{T}_{\text {eeg } \rightarrow \text { ecg }}$ and $\mathrm{T}_{\text {ecg } \rightarrow \text { eeg }}$ in first stage of non-rapid eye movement sleep. in this figure, $\mathrm{x} 1$

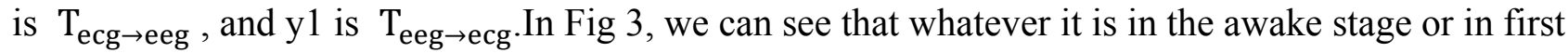
stage of non-rapid eye movement sleep, the symbolic transfer entropy $\mathrm{T}_{\text {eeg } \rightarrow \text { ecg }}$ is greater than

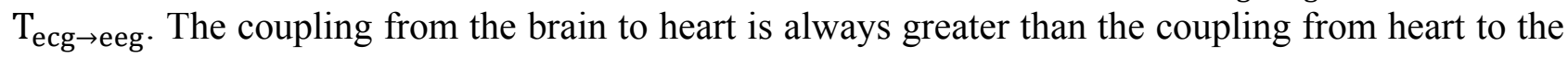
brain.

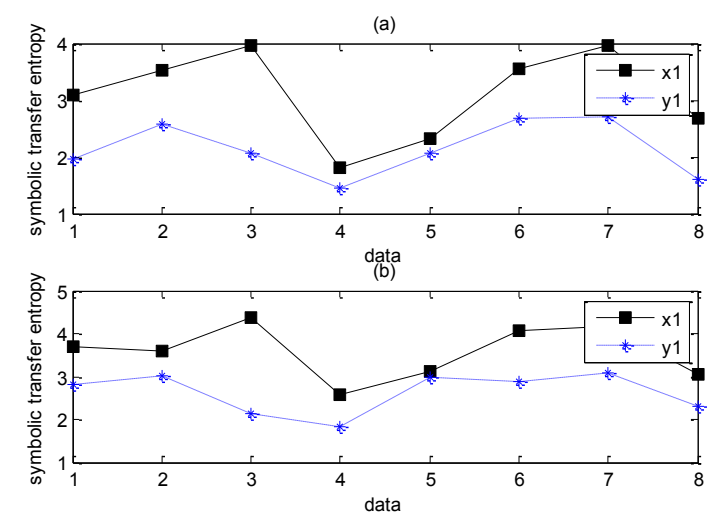

Fig. 4. Comparison of symbolic transfer entropy based on permutation entropy symbolization in awake stage and in first stage of non-rapid eye movement sleep.

Fig 4(a) is the comparison of symbolic transfer entropy $\mathrm{T}_{\text {ecg } \rightarrow \text { eeg }}$ in awake stage and in first stage of non-rapid eye movement sleep and Fig 4(b) is the comparison of symbolic transfer entropy $\mathrm{T}_{\text {eeg } \rightarrow \text { ecg }}$ in awake stage and in first stage of non-rapid eye movement sleep. in this figure, $\mathrm{x} 1$ is the symbolic transfer entropy in awake stage, and y1 is in first stage of non-rapid eye movement sleep. In figure 4 , whatever it is $\mathrm{T}_{\text {eeg } \rightarrow \text { ecg }}$ and $\mathrm{T}_{\text {ecg } \rightarrow \text { eeg }}$, we can see that the value of the awake stage is always greater than the value of first stage of non-rapid eye movement sleep. This suggests that 
both the coupling from brain to the heart and from heart to the brain, the symbolic transfer entropy in awake stage is larger than first stage of non-rapid eye movement sleep.

\subsection{The Symbolic transfer entropy based on quantization $T_{e c g \rightarrow e e g}$ and $T_{e e g \rightarrow e c g}$ are compared between awake stage and first stage of non-rapid eye movement sleep.}
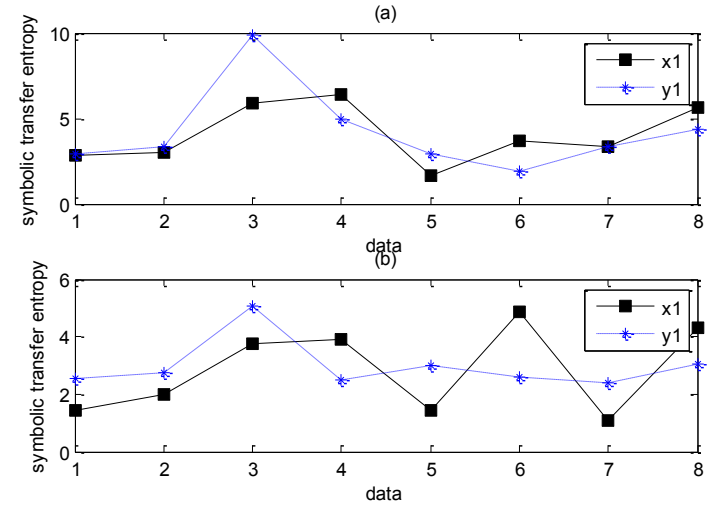

Fig. 5. Comparison of symbolic transfer entropy based on quantization in awake stage and in first stage of non-rapid eye movement sleep.

Fig 5(a) is the comparison $\mathrm{T}_{\text {eeg } \rightarrow \text { ecg }}$ and $\mathrm{T}_{\text {ecg } \rightarrow \text { eeg }}$ in awake stage and Fig 5(b) is the comparison $\mathrm{T}_{\text {eeg } \rightarrow \text { ecg }}$ and $\mathrm{T}_{\text {ecg } \rightarrow \text { eeg }}$ in first stage of non-rapid eye movement sleep. in this figure, $\mathrm{x} 1$ is $\mathrm{T}_{\text {ecg } \rightarrow \text { eeg }}$,

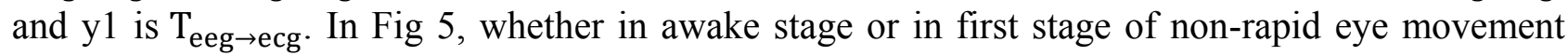
sleep, the value of $\mathrm{T}_{\text {eeg } \rightarrow \text { ecg }}$ is larger than $\mathrm{T}_{\mathrm{ecg} \rightarrow \mathrm{eeg}}$, but the conclusion is not obvious, there is only such a trend.
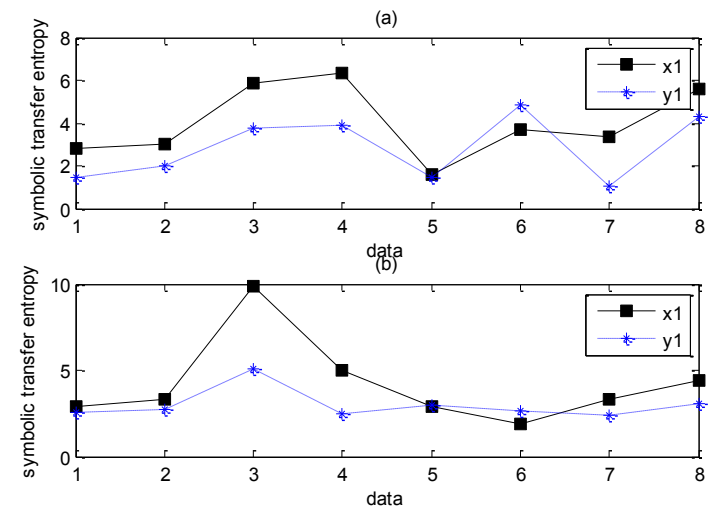

Fig. 6. Comparison of symbolic transfer entropy based on quantization in awake stage and in first stage of non-rapid eye movement sleep.

Fig 6(a) is the comparison of symbolic transfer entropy $\mathrm{T}_{\text {ecg } \rightarrow \text { eeg }}$ in awake stage and in first stage of non-rapid eye movement sleep and Fig 6(b) is the comparison of symbolic transfer entropy $\mathrm{T}_{\text {eeg } \rightarrow \text { ecg }}$ in awake stage and in first stage of non-rapid eye movement sleep; in this figure, $\mathrm{x} 1$ is the symbolic transfer entropy in awake stage, and y1 is in first stage of non-rapid eye movement sleep. In figure 6 , whatever $\mathrm{T}_{\text {eeg } \rightarrow \text { ecg }}$ and $\mathrm{T}_{\mathrm{ecg} \rightarrow \mathrm{eeg}}$, The value in awake stage is always greater than the value in in first stage of non-rapid eye movement sleep. this conclusion is not true for all data, but just a trend.

\subsection{A detailed analysis of the experimental result}

From the above analysis, using symbolic transfer entropy method based on permutation entropy symbolization, we can conclude that $\mathrm{T}_{\text {eeg } \rightarrow \text { ecg }}$ is larger than $\mathrm{T}_{\text {ecg } \rightarrow \text { eeg }}$ whatever in awake stage and in first stage of non-rapid eye movement sleep. this show both in awake stage and in first stage of non-rapid eye movement sleep, the coupling from brain to heart is larger than from heart to brain. In 
this case, it is also concluded that the symbolic transfer entropy value of awake stage is larger than first stage of non-rapid eye movement sleep, whatever $\mathrm{T}_{\text {eeg } \rightarrow \text { ecg }}$ or $\mathrm{T}_{\mathrm{ecg} \rightarrow \mathrm{eeg}}$. This suggest that the coupling between the heart and the brain in first stage of non-rapid eye movement sleep is less than the coupling between heart and the brain in awake stage. Using symbolic transfer entropy based on permutation entropy symbolization can drawn above conclusion. But the above-mentioned conclusion can not be obtained by using the symbolic transfer entropy based on quantization, the above conclusion are not all satisfied, and compare with the symbolic transfer entropy based on permutation entropy symbolization, symbolic transfer entropy based on quantization is more volatile and more unstable. This suggests that when analyze sleep signal, using symbolic transfer entropy method based on permutation entropy symbolization is better.

Table1-Table4 are specific data for experimental result, and "Quantify" represent the value of the symbolic transfer entropy based on the quantization, " $\tau=1$ " and " $\tau=5$ " all represent the value of the symbolic transfer entropy based on permutation entropy symbolization, and $\tau=1$ represent time delay is one, $\tau=5$ represents the time delay is five.

Table 1 symbolic transfer entropy $\mathrm{T}_{\text {ecg } \rightarrow \text { eeg }}$ in awake stage

\begin{tabular}{|l|l|l|l|l|l|l|l|l|}
\hline & Slp41 & Slp45 & Slp48 & Slp59 & Slp411 & Slp451 & Slp481 & Slp591 \\
\hline Quantify & 2.8368 & 3.03 & 2.3396 & 6.3701 & 1.6219 & 3.662 & 3.3609 & 5.6256 \\
\hline$\tau=1$ & 3.0963 & 3.5171 & 3.9575 & 1.8119 & 2.3083 & 3.5622 & 3.9652 & 2.6743 \\
\hline$\tau=5$ & 7.0811 & 7.1897 & 5.8876 & 5.1513 & 2.5962 & 6.1421 & 8.2208 & 6.4749 \\
\hline
\end{tabular}

Table 2 symbolic transfer entropy $\mathrm{T}_{\text {eeg } \rightarrow \text { ecg }}$ in awake stage

\begin{tabular}{|c|c|c|c|c|c|c|c|c|}
\hline & Slp41 & Slp45 & Slp48 & Slp59 & Slp411 & Slp451 & Slp481 & SIp591 \\
\hline Quantify & 2.9138 & 3.3302 & 4.8744 & 4.9675 & 2.8919 & 1.8871 & 3.3558 & 4.3959 \\
\hline$\tau=1$ & 3.6781 & 3.6059 & 4.3687 & 2.5891 & 3.1069 & 4.081 & 4.1764 & 3.0496 \\
\hline$\tau=5$ & 8.4982 & 8.1666 & 9.8972 & 4.569 & 5.6463 & 8.2112 & 10.201 & 5.3442 \\
\hline
\end{tabular}

Table 3 symbolic transfer entropy $\mathrm{T}_{\text {ecg } \rightarrow \text { eeg }}$ in first stage of non-rapid eye movement sleep

\begin{tabular}{|c|c|c|c|c|c|c|c|c|}
\hline & Slp41 & Slp45 & Slp48 & Slp59 & SIp411 & Slp451 & Slp481 & SIp591 \\
\hline Quantify & 1.4282 & 2.0087 & 1.2846 & 3.9208 & 1.4246 & 4.8426 & 1.0702 & 4.2859 \\
\hline$\tau=1$ & 1.967 & 2.5854 & 2.0572 & 1.4443 & 2.0652 & 2.6892 & 2.6978 & 1.611 \\
\hline$\tau=5$ & 2.4361 & 3.8138 & 3.7329 & 5.3224 & 2.6993 & 3.8348 & 3.7015 & 5.1414 \\
\hline
\end{tabular}

Table 4 symbolic transfer entropy $\mathrm{T}_{\mathrm{eeg} \rightarrow \mathrm{ecg}}$ in first stage of non-rapid eye movement sleep

\begin{tabular}{|c|c|c|c|c|c|c|c|c|}
\hline & Slp41 & Slp45 & Slp48 & Slp59 & SIp411 & Slp451 & Slp481 & SIp591 \\
\hline Quantify & 2.5488 & 2.7371 & 2.3151 & 2.5119 & 2.9983 & 2.6108 & 2.3985 & 3.0443 \\
\hline$\tau=1$ & 2.8226 & 3.0259 & 2.143 & 1.8141 & 2.9805 & 2.8711 & 3.0987 & 2.2898 \\
\hline$\tau=5$ & 5.4648 & 5.083 & 5.0921 & 3.9198 & 5.9846 & 5.9174 & 6.6542 & 4.3103 \\
\hline
\end{tabular}

\section{Conclusion}

On the basic research of symbolic transfer entropy based on quantization, this paper adopts the method of symbolic transfer entropy based on permutation entropy symbolization to analyze sleep signal. Through theoretical and experimental analysis, it found that when analyzing the coupling of EEG and ECG sleeping signal, compare with symbolic transfer entropy based on quantization, the symbolic transfer entropy method based on permutation entropy symbolization can obtain better results. 
In the analysis of data, symbolic transfer entropy method based on quantization is a static division method, quantify data directly, which will ignore the details of the sequence information, so the experimental results will be greatly affected. In this paper, the method of permutation entropy symbolization is used to symbolize by using the correlation between the sequences, and the detailed information of the sequence is preserved. The experimental result show that in analysis of sleep signal, the result of symbolic transfer entropy method based on permutation entropy symbolization is better than the method based on quantization. And this result suggest that the method based on permutation entropy symbolization is better for analyzing the sleep signal, it can retain the details of sequence information, and more conductive to clinical applications.

\section{Acknowledgement}

The project is supported by the National Natural Science Foundation of China (Grant Nos. 61271082, 31671006), Jiangsu Provincial Key R \& D Program (Social Development) (Grant No.BE2015700, BE2016773), the Natural Science Foundation of Jiangsu Province (Grant No. BK20141432),Natural Science Research Major Program in Universities of Jiangsu Province (Grant No.16KJA310002).

\section{References}

[1] Dliou A, Latif R, Laaboubi M. Arrhythmia ECG signal analysis using non parametric timefrequency techniques[J]. International Journal of Computer Applications, 2012, 41(4).

[2] Rai H M, Trivedi A, Shukla S. ECG signal processing for abnormalities detection using multiresolution wavelet transform and Artificial Neural Network classifier[J]. Measurement, 2013, 46(9): 3238-3246.

[3] Owis M I, Abou-Zied A H, Youssef A B M. Study of features based on nonlinear dynamical modeling in ECG arrhythmia detection and classification[J]. IEEE transactions on Biomedical Engineering, 2002, 49(7): 733-736.

[4] Iriarte J, Urrestarazu E, Valencia M. Independent component analysis as a tool to eliminate artifacts in EEG: a quantitative study[J]. Journal of clinical neurophysiology, 2003, 20(4): 249-257.

[5] Staniek M, Lehnertz K. Symbolic transfer entropy[J]. Physical Review Letters, 2008, 100(15): 158101.

[6] Staniek M, Lehnertz K. Symbolic transfer entropy: inferring directionality in biosignals[J]. Biomedizinische Technik/Biomedical Engineering, 2009, 54(6): 323-328.

[7] Bandt C, Pompe B. Permutation entropy: a natural complexity measure for time series[J]. Physical review letters, 2002, 88(17): 174102.

[8] Zanin M, Zunino L, Rosso O A, et al. Permutation entropy and its main biomedical and econophysics applications: a review[J]. Entropy, 2012, 14(8): 1553-1577.

[9] Xiaoru Jing, Yanting $\mathrm{Hu}$, Jun Wang,et al. Symbolic transfer entropy analysis of sleep stage classification[J]. Beijing Biomedical Engineering, 2012, 31(4):372-376. 